\title{
Erratum: Serine/threonine Kinases Play Important Roles in Regulating Polyunsaturated Fatty Acid Biosynthesis in Synechocystis sp. PCC6803
}

\author{
Frontiers Production Office* \\ Frontiers Media SA, Lausanne, Switzerland
}

Keywords: microalgae, serine/threonine kinase system, polyunsaturated fatty acids, biosynthesis, Synechocystis sp. PCC6803

\section{An Erratum on}

OPEN ACCESS

Approved by:

Frontiers Editorial Office,

Frontiers Media SA, Switzerland

*Correspondence:

Frontiers Production Office

production.office@frontiersin.org

Specialty section:

This article was submitted to

Bioprocess Engineering,

a section of the journal

Frontiers in Bioengineering and

Biotechnology

Received: 26 March 2021

Accepted: 26 March 2021

Published: 19 April 2021

Citation:

Frontiers Production Office (2021)

Erratum: Serine/threonine Kinases

Play Important Roles in Regulating

Polyunsaturated Fatty Acid

Biosynthesis in Synechocystis sp.

PCC6803.

Front. Bioeng. Biotechnol. 9:686089.

doi: 10.3389/fbioe.2021.686089
Serine/threonine Kinases Play Important Roles in Regulating Polyunsaturated Fatty Acid Biosynthesis in Synechocystis sp. PCC6803

by Chen, G., Cao, Y., Zhong, H., Wang, X., Li, Y., Cui, X., et al. (2021). Front. Bioeng. Biotechnol. 9:618969. doi: 10.3389/fbioe.2021.618969

Due to a production error, there was a mistake in the caption of Figure 3 as published. The corrected caption appears below.

Figure 3. Changes in serine/threonine kinase (STK) gene expression in wild type and mutant strains detected after different periods of exposure to normal light. WT represents wild type Synechocystis sp. PCC6803; spkD- represents the spkD knockout mutant; spkG- represents the $s p k G$ knockout mutant. The experiment was carried out under a normal light intensity of 40 $\mu \mathrm{mol} \cdot \mathrm{m}^{-2} \cdot \mathrm{s}^{-1}$. (A-E) show the relative expression levels of $s p k A, s p k B, s p k C, s p k F$, and $s p k E$, respectively, in the wild type and two mutant strains. (F) Relative expression levels of $s p k G$ in the wild type and $s p k D$-. The data correspond to the left vertical axis. The black bars represent the wild type. Synechocystis sp. PCC6803, and the red bars represent the mutation that knocked out spkD. The right vertical axis shows the relative expression levels of $s p k D$ in the wild type and $s p k G^{-}$, and the black bar represents the wild type. The blue bar represents the mutation that knocked out spkG. Values are means \pm SD (bars) of three independent experiments conducted on different days. The absence of a bar indicates that the SD falls within the symbol.

The publisher apologizes for this mistake. The original article has been updated.

Copyright $(02021$ Frontiers Production Office. This is an open-access article distributed under the terms of the Creative Commons Attribution License (CC BY). The use, distribution or reproduction in other forums is permitted, provided the original author(s) and the copyright owner(s) are credited and that the original publication in this journal is cited, in accordance with accepted academic practice. No use, distribution or reproduction is permitted which does not comply with these terms. 